\title{
Extracting Features from Tactile Maps
}

\author{
Parris S. Wellman and Robert D. Howe \\ Division of Engineering and Applied Science \\ Harvard University, Cambridge, MA 02138 \\ parris@hrl.harvard.edu,howe@ deas.harvard.edu
}

\begin{abstract}
Tactile imaging is a newly developed mechanical sensing technology for documenting the properties of hard lumps contained in soft tissue. An examiner strokes a scan head across tissue that contains a mass and images of the distributed contact pressure between the head and the tissue are recorded. We have developed models that predict these pressure distributions from geometric and material properties. We then use inversion algorithms developed from these models to extract lump size and shape. In a limited clinical trial on 24 surgical patients, lump size was estimated with less than $17 \%$ mean absolute error when compared with ex-vivo size measurements. This is more than twice as accurate as either clinical breast examination or ultrasound examination of the same lumps. This result demonstrates that tactile imaging has the potential to improve the accuracy of clinical breast examination.
\end{abstract}

\section{Introduction}

The sense of touch is an invaluable and widely applied clinical tool, particularly for the detection and diagnosis of breast cancer, where a palpable lump is the most common symptom of the disease [1]. Palpation often plays a primary role in monitoring benign breast lumps for change as they are often not visible on mammograms or in ultrasound images because of breast density or scarring. In these cases, $\mathrm{CBE}$ is the most effective tool for monitoring a breast for changes in time. The problem with this method is that it is difficult to accurately verbalize and record tactile sensations, prompting one physician to remark "I can only deplore tumor size expressed in terms of fruits, nuts, or vegetables, but ... medical students ... continue to prefer these agricultural analogies" [2]. Even assessing the size of a lump can be problematic, as one study reports that nearly $100 \%$ change in the size of a lump is required for it to considered a noticeable clinical change [3]. A method for accurately recording tactile sensations from physical exams would make CBE more objective, and could greatly improve the ability to detect changes.

Many new breast examination technologies have been proposed but the typical intended application has most often been screening, where the primary challenge is the detection of the presence of a lump in the breast [4,5,6,7]. In contrast, the monitoring application we describe assumes that the lump has been detected, and the challenge is to accurately document parameters such as lump size, shape, and hardness. Sarvazyan [8] has proposed a method that uses distributed pressure measurement to estimate the properties of breast and prostate lumps. He uses an iterative solution of a finite element model in order to estimate the properties of these 
lumps but has only demonstrated it in the prostate. Several new imaging modalities also show promise for characterizing breast lumps, including magnetic resonance and ultrasound elastography $[9,10]$. However, these techniques require elaborate and relatively expensive imaging systems and skilled technicians.

This paper reports the development of a system that uses simple algorithms for extracting breast lump features from measurements of contact pressure. The clinician strokes a "scan head" containing an array of pressure sensors over the breast. Signal processing algorithms assemble a "tactile map" of the breast and estimate lump parameters such as size and shape. The goal is to provide objective and repeatable documentation of palpation information in a form that is easily understood by the clinician and even the patient. These images may be incorporated into the patient record as a means of tracking lump changes across time. The technique is designed to be inexpensive, noninvasive, quick, and easy to use. It will enable follow-up examinations to be conducted frequently with minimal risk and inconvenience.

In subsequent sections we discuss mathematical forward models that relate geometric and material properties of the breast to pressure distributions measured on the surface of the scan head. We use these forward models to develop inversion algorithms that can be used to extract the size of palpable lumps. Finally we discuss the system performance in a limited clinical trial of 24 breast surgery patients.

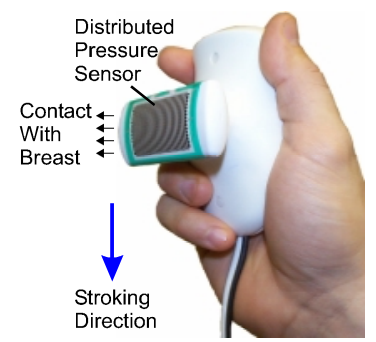

(a)

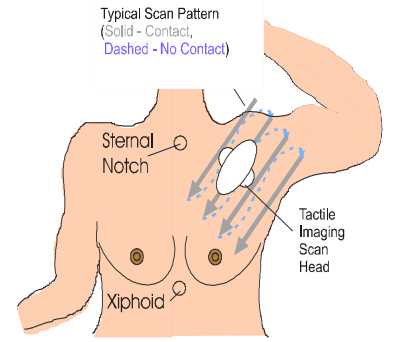

(b)

Fig. 1. - (a) The scan head of the tactile imaging system. The indentor portion of the scan head is an array of piezoresistive pressure sensors (16 rows by 26 columns, $1.5 \mathrm{~mm}$ spacing). A magnetic position tracker is located in the head. (b) The patient is placed supine with the ipsilateral arm over the head to stabilize the breast and minimize tissue thickness. The clinician begins the examination by indicating the location of the readily palpable sternal notch and xiphoid to facilitate comparison of follow-up examinations. The clinician then presses the indentor into the breast near the mass, and strokes the sensor repeatedly over the area of the mass and its immediate surroundings. The skin surface is lubricated to minimize friction.

\subsection{System Description}

Figure 1(a) shows the tactile imaging system "scan head" that the physician strokes over the breast. The array of piezoresistive pressure sensors mounted on its surface have a range of $0-34 \mathrm{kPa}$ and are calibrated before each use using a pressure bladder. A magnetic tracker in the handle senses the relative position and rotation of each pressure image. A computer samples the tracker and pressure sensor array every 5 milliseconds. The tactile mapping algorithm assembles these individual pressure frames, or tactile images, to form a composite tactile map of the mass. 


\section{Methods}

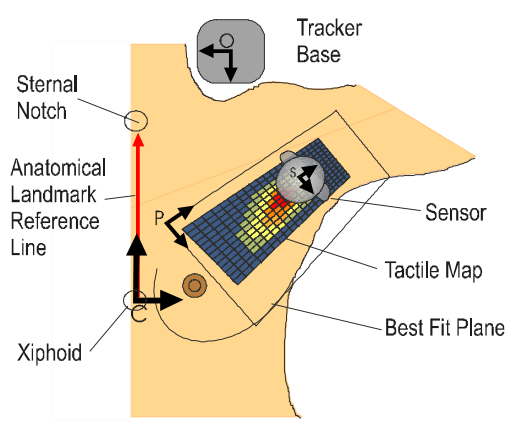

(a)

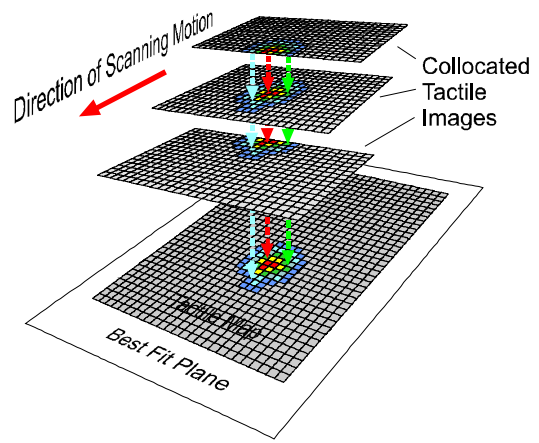

(b)

Fig. 2 -(a) A tactile map is registered to the patient using coordinate frame $\mathrm{C}$, determined by the sternal notch and xiphoid. Frame $\mathrm{S}$ is the location of the scan head during each image, frame $\mathrm{P}$ is the best-fit plane frame and Frame $\mathrm{O}$ is the base frame for the position tracker. (b) $\mathrm{A}$ tactile map is created by collocating each image, projecting and averaging the values onto the plane that best fits the sensor motion in the neighborhood of the mass.

The shape of the indentor was designed to provide a nearly uniform pressure distribution when pressed into homogeneous tissue [11], to minimize the dynamic range required for the pressure sensors and to simplify the signal processing. Analysis shows that this shape is nearly a section of a circular cylinder, but the exact shape depends on the tissue stiffness and the indentation pressure applied by the user [12]. Indentor size was chosen to require a comfortable range of applied force. Finite element analysis showed that for normal breast tissue $(15-25 \mathrm{kPa}$ modulus), [13] and anticipated user-applied pressures $(5-14 \mathrm{kPa})$, the pressure variation across the indentor is within $7.5 \%$ of mean.

Clinician stroking technique and patient placement must be controlled in order to obtain repeatable results; typical technique is shown in Figure 1(b). An audible tone assists the clinician in maintaining the desired average pressure range while the breast is lubricated to reduce friction. The clinician continues stroking until the tactile map, created in real time on the monitor, reflects the palpable extent of the mass. The tactile map combines the pressure images in a form that is readily interpreted. It also averages multiple images to reduce noise from the transducer, technique variations and small mass motions. Just before making a tactile map, the examiner indicates the sternal notch and the xiphoid using the scan head as shown in Figure 2(a). This provides an alignment reference for future examinations of the patient. Producing a tactile map from the scans made by the examiner can be summarized in three steps

i. Determine the best-fit plane on which to project the data from the motions of the examiner in the neighborhood of the lump.

ii. Eliminate pressure frames outside the pressure range of interest and normalize the remainder using the average of the frame to account for the remaining variation in pressure level.

iii. Collocate and average the frames on the best-fit plane to form the composite tactile map as shown in Figure 2(b). 
Once the maps are constructed, we need to use inversion algorithms to extract lump features. To develop these algorithms we first develop forward models that predict the pressure distribution given the geometric and material properties. We use these models to develop inversion algorithms.

\subsection{Forward Models}

The most common form of cancer is infiltrating ductal carcinoma [14] which forms in the lactiferous ducts and then spreads through the tissue layers. In our observation these lumps, as well as other non-cancerous masses, become palpable when they project into the fat layer overlying the glandular tissue. We consider the breast to be a three-layer model: a layer of fat, a layer of normal glandular tissue and the rigid chest wall, as shown in Figure 3(a) with a single palpable mass that forms at the boundary of the glandular tissue and fat. We also assume that the tissue is elastic and isotropic, and like other biological tissue is nearly incompressible [15]. Other researchers have solved various aspects of this problem $[16,17,18,19]$ but no analytic solution exists for the full case presented here. We use a finite element model with geometry in Figure 3(a) to determine the relationship of these properties to the output pressure distribution. Because the indentor is much longer than it is wide, see Figure 1(a), we solve the problem with a two-dimensional plane strain model. This model is sufficient because we are only looking for trends to develop inversion algorithms.

We assume that the indentor and chest wall are rigid, the normal glandular tissue is ten times as stiff as the surrounding fat, and the fat has an elastic modulus of $5 \mathrm{kPa}$, based on our measurements of the elastic properties of breast tissues [20]. Most palpable masses exhibit a nonlinear relationship between stress and strain so we will examine the effect of this change in modulus by varying the ratio of the tumor stiffness to the surrounding tissue stiffness. The indentor is pressed into the tissue under constant force as it slides across the surface.

It is also desirable to have an analytical expression that predicts the pressure at any point on the surface of the indentor as a function of its location, the geometric and material properties. We represent the indentor face pressure distribution for each location of the indentor along the surface of the tissue as the weighted sum of two pressure distributions. The first, $P_{l}$ is the pressure distribution across the face of the indentor that would be produced if there were no lump present. The second, $P_{2}$, is the difference between the pressure distribution far from the lump, $P_{1}$ and $P_{0}$, which is the pressure distribution on the face of the indentor when it is centered on the lump. Figure 3(b) shows these distributions, where $P_{2}=P_{0}-\alpha P_{1}$ and $\alpha=0.5$ is an arbitrary parameter chosen to be sure that $P_{2}>0$.

In Figure 4(a) we see that there is some variation in the shape of the pressure distribution far from the lump that depends upon the thickness of the tissue. We use a model which is motivated by Hertz' classic solution [21]

$$
P_{1}(s, t)=\left\{\begin{array}{cc}
P_{O}(t) \cdot\left(1-\left(\frac{s}{a(t)}\right)^{2}\right)^{\frac{1}{2}} & -w \leq s \leq w \\
0 & \text { otherwise }
\end{array}\right.
$$


where $2 w$ is the width of the head $(2.4 \mathrm{~cm}), P_{o}(t)$ and $a(t)$ were determined by fitting the curves in Figure 4(a) with Equation 1. The curves in Figure 4(b) can be fit with a Gaussian distribution that is a function of location along the surface of the tissue, $x$, lump diameter, $d$, stiffness ratio of tumor to surrounding fat $E_{2} / E_{l}$, and depth ratio from the surface of the tissue to the center of the lump, $h / d$, as,

$$
P_{2}\left(x, d, E_{2} / E_{1}, h / d\right)=g\left(d, E_{2} / E_{1}, h / d\right) \cdot e^{-\left(\frac{x^{2}}{2 \sigma\left(d, E_{2} / E_{1}, h / d\right)^{2}}\right)}
$$

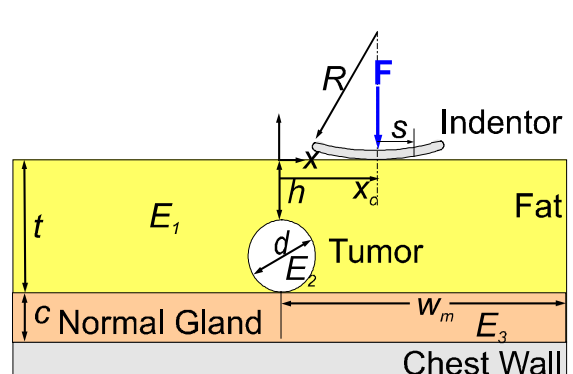

(a)

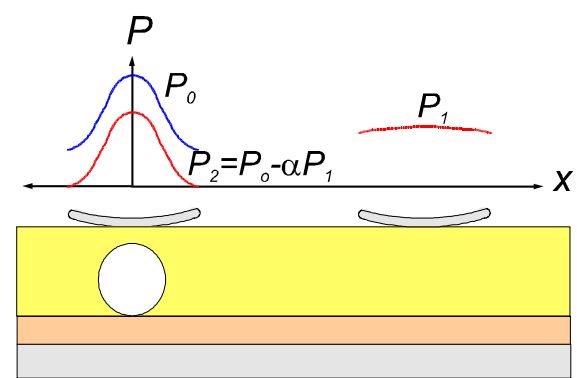

(b)

Fig. 3 - (a) The geometry of the plane strain model which has been idealized to contain only a single focal mass and three layers of tissue. (b) The pressure distributions modeled in the empirical fit.

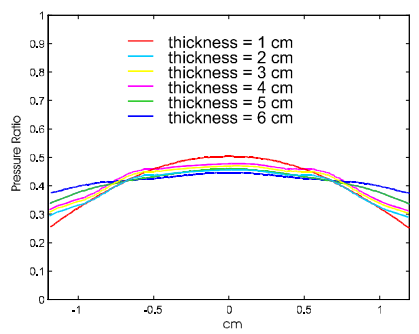

(a)

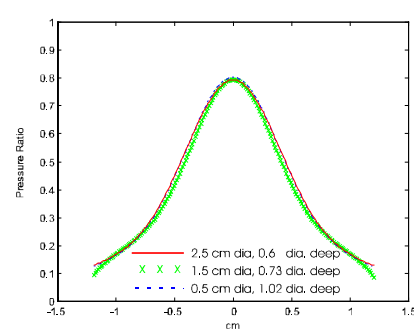

(b)

Fig. 4 - (a) Pressure frames, from finite element models, FEM, for size different tissue thickness, $P_{l}$, on the face of the indentor with the center of the indentor far from the lump. (b) Pressure frames (FEM), $P_{0}$, with the indentor centered on the lump for 3 different cases.

We must also develop a two dimensional analog to the tactile map. A tactile map is nothing but the spatial average of all pressure values for a given set of pressure frames. We can also view this average as the average of all pressure curves formed by an individual element on the face of the indentor as it slides across the surface. We change variables to $s=x-x_{c}$ and write each of these curves as

$$
P_{\text {surface }}\left(x, s, d, E_{2} / E_{1}, h / d\right)=P_{2}\left(x, d, E_{2} / E_{1}, h / d\right) \cdot\left(\frac{P_{1}(s, t)}{\max \left(P_{1}(s, t)\right)}\right)^{2}+\kappa\left(x_{c}, d\right) \cdot P_{1}(s, t)
$$


where the $P_{1}^{2}$ term a good fit to the finite element model data as the indentor slides off of the lump, $x-x_{c}$ is restricted to the head width, and

$$
\kappa\left(x_{c}, d\right)=\left\{\begin{array}{cc}
0.5 \cdot\left(1+\frac{8}{3 d}\left|x_{c}\right|\right) & 0 \leq\left|x_{c}\right| \leq \frac{8}{3} d \\
1 & \text { otherwise }
\end{array}\right.
$$

The equation for the map is just the average of all of the curves. We evaluate this expression to reveal that the map is Gaussian, with the same standard deviation as equation 2 and different amplitude.

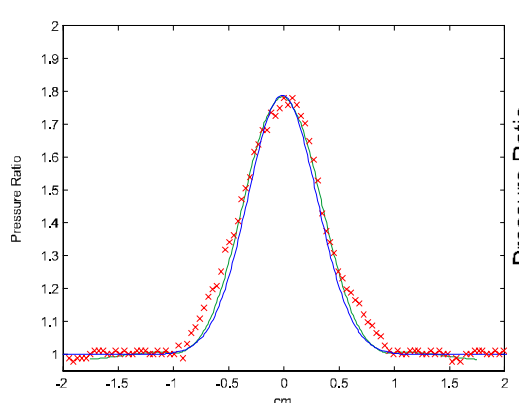

(a)

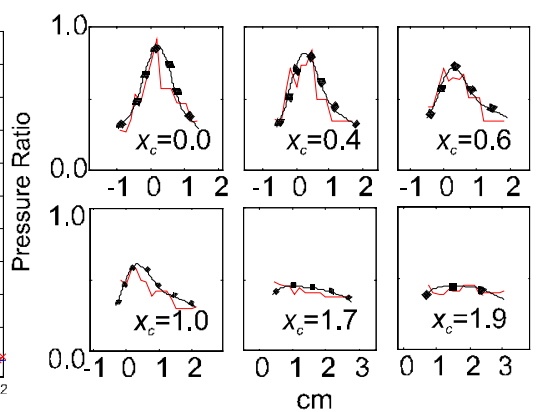

(b)

Fig. 5 - (a) Comparison for same geometry of the centerline of the tactile map and the two dimensional maps from finite element and empirical models $(\mathrm{x}$ - centerline of map, solid line FEA model, dotted line - empirical model). There is less than a $2.6 \%$ mean squared difference between the three curves. (b) Individual pressure frames for a silicone rubber breast model with a $1.25 \mathrm{~cm}$ diameter, 0.825 diameter deep rigid spherical inclusion (dotted = empirical model, solid $=$ FEA model, thick solid $=$ actual data).

\subsection{Forward Model Validation}

In order to verify that the finite element and empirical models give equivalent results, a set of trials on four different silicone rubber breast models $(\mathrm{d}=1.25 \mathrm{~cm}$, $\mathrm{h} / \mathrm{d}=0.825 ; \mathrm{d}=1.55 \mathrm{~cm}, \mathrm{~h} / \mathrm{d}=1.0 ; \mathrm{d}=1.55 \mathrm{~cm}, \mathrm{~h} / \mathrm{d}=1.4 ;$ and $\mathrm{d}=1.875 \mathrm{~cm}, \mathrm{~h} / \mathrm{d}=1.2$ ) were conducted. The pressure frames and tactile maps were compared along the centerline of the indentor to the model derived results. Figure 5(a) shows an illustrative result. The average mean square difference between the model frames and the real data frames is less than $15 \%$, primarily because of the noise in the real data.

To be sure that the empirical model accurately reflects the finite element results, they were compared on a frame by frame level. Figure 5(b) shows a representative set of frame by frame pressure curves for four lumps. The tests spanned a range of lump sizes from $0.5 \mathrm{~cm}$ to $2.5 \mathrm{~cm}$ in diameter, and depth ratios $h / d$ from 0.6 to 1.8 diameters. Table 1 summarizes the mean squared error figures for ten cases. The average mean squared difference for these frames is less than $4.2 \%$, while the average for all frames in a map it is $4.3 \%$. The finite element and empirical models were also compared on the map level, and Table 2 summarizes the results. 
Table 1 - Mean squared difference, MSD, between the empirical model and the finite element models for the indentor frames centered on the lump. Average MSD $=4.3 \%$.

\begin{tabular}{|l|l|l|l|l|l|}
\hline$h / d$ & $d=0.5 \mathrm{~cm}$ & $d=1.0$ & $d=1.5$ & $d=2.0$ & $d=2.5$ \\
\hline 0.6 & $4.1 \%$ & $4.3 \%$ & $4.1 \%$ & $4.1 \%$ & $4.2 \%$ \\
\hline 1.8 & $4.1 \%$ & $4.2 \%$ & $4.2 \%$ & $4.0 \%$ & $4.1 \%$ \\
\hline
\end{tabular}

Table 2 - Mean squared difference between the empirical model and the finite element models for the composite maps. Average MSD $=2.5 \%$.

\begin{tabular}{|l|l|l|l|l|l|}
\hline$h / d$ & $d=0.5$ & $d=1.0$ & $d=1.5$ & $d=2.0$ & $d=2.5$ \\
\hline 0.6 & $2.4 \%$ & $2.7 \%$ & $2.5 \%$ & $2.3 \%$ & $2.2 \%$ \\
\hline 1.8 & $2.3 \%$ & $2.5 \%$ & $2.4 \%$ & $2.5 \%$ & $2.6 \%$ \\
\hline
\end{tabular}

\subsection{Inverse Models}

Because the rubber model data and the finite element and empirical models showed good agreement in the test cases, we ran a larger set of models to determine the trends that would be present in the tactile maps. These tests spanned all possible combinations of mass diameters $0.5,1.0,1.5,2.0$ and $2.5 \mathrm{~cm}$, mass depths of $0.6,0.8$, 1.0, 1.2, 1.5 and 2.0 diameters, and mass stiffness to fat stiffness ratios of 2,5,8,10 and 100 times. These maps are Gaussian and can be fit with

$$
P_{\text {map }}(x)=a \cdot e^{-\left(x^{2} / 2 \sigma\right)}+1 \text {. }
$$

There are some combinations of lump size and depth that lead to identical output pressure distributions, for a given stiffness ratio. More cases overlap if the lump stiffness is varied. However, Figure 6 makes it clear that the stiffness of the lump makes very little difference to the width of the pressure distribution for lumps greater than eight times as stiff as the fat. Fortunately, our measurements of the elastic properties of breast lumps show that they are at least eight times as stiff as the fat tissue [22]. We ignore the distribution width variations because of stiffness and assume that all lumps are hard $\left(\mathrm{E}_{2} / \mathrm{E}_{1}=100\right)$.

The models reveal that the width of the distribution increases with lump depth and also show that the percent change in width of the pressure distribution (referenced to 1 diameter deep) can be fit with the allotropic relationship, $\Delta \sigma=e a^{f}$ where $e$ and $f$ are real numbers, and is shown in Figure 7(a). Figure 7(b) reveals that $\sigma$ is linearly related to the lump diameter at one diameter depth. Therefore

$$
d=m \cdot(\sigma / \Delta \sigma)+b .
$$

In order to extend this inversion algorithm to the full tactile map, we observe that the three dimensional maps created with the real system also appear to be Gaussian and we minimize the squared error between the map and

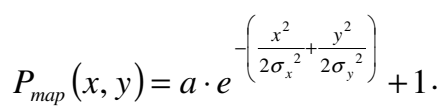

We use Equation 6 to determine the two diameters, $d_{x}$ and $d_{y}$ of the lump from the fit. 


\section{Clinical Testing}

To test the hypothesis that tactile imaging is more accurate than clinical breast examination (CBE) or ultrasound at assessing size, a limited trial involving surgery patients was performed. We compared size estimates from the various methods with accurate size measurements of the masses after excision. After informed consent was obtained, subjects received mammograms, ultrasound examinations and CBEs following the usual course of treatment. One of the two surgeons in the study made three to five tactile maps of each mass prior to surgery using different stroking techniques. Maximum and minimum sizes were estimated in each of the maps as described above. The maximum size from CBE and ultrasound were recorded. After excision, the mass was bisected parallel to the plane of the tactile map and the palpable extents were measured using a caliper. One African-American and twentythree Caucasian women participated in the study; ages ranged from 39 to 84 years old. Patients presented a total of 19 infiltrating ductal cancers, 2 fibroadenomas, 1 lobular carcinoma, 1 Phyllodes tumor, 1 papilloma and 1 patient with fibrotic adipose tissue.

Figure 8(a) shows an illustrative tactile map of an infiltrating ductal carcinoma and Figure 8(b) shows a photograph after surgical excision and bisection. Figure 9 presents a comparison of the maximum size estimates from ultrasound, CBE and tactile imaging, respectively, to the ex vivo size measurements made in the clinic. The average standard deviation of size estimated from multiple maps for a single mass is $15 \%(2.6 \mathrm{~mm})$. The percent mean absolute error (MAE) between ultrasound and ex vivo measurements is $34 \%$, for CBE it is $47 \%$ and for tactile imaging it is $17 \%$, which makes tactile imaging more than twice as accurate as either clinical breast examination or ultrasound using this inversion algorithm. The slope of the best fit line with zero intercept for maximum size estimates from tactile imaging as compared to ex vivo size measurements is $1.04\left(\mathrm{r}^{2}=0.64\right)$, while for CBE it is 1.26 $\left(r^{2}=0.39\right)$ and for ultrasound it is $0.90\left(r^{2}=0.14\right)$. We have not presented size information from the mammograms, since only the presence or absence of a mass was typically reported. Of the 25 masses, 24 were visible in mammograms, one was not palpable and one was not visible on ultrasound.

\section{Conclusions and Future Work}

The inversion algorithms presented here performed well in clinical tests made on real breast lumps, and provided accuracy that was at least twice as accurate as either clinical breast examination or ultrasound breast examination. This validates that the model is useful to obtain results in the real clinical situation, and confirms that we have taken an appropriate approach. There are many improvements that could be made to the model, including elliptical rather than spherical lump geometry, mobile rather than well fixed lumps and non-linear tissue stiffness. Non-linear stiffness is important because it has been shown that tissue stiffness, and the change in tissue stiffness with strain are related to histological diagnosis [23,24]. These enhanced models could make it possible to develop feature extraction algorithms that enhance the utility of tactile imaging by taking it from a documentary to a diagnostic role. 


\section{Acknowledgements}

We gratefully acknowledge the support of the Whitaker Foundation and Assurance Medical. We thank the entire team at Assurance Medical who made this project possible. We would also like to thank Drs. Edward Dalton, Kenneth Kern, Matthew Freedman, David Krag and Prof. Eric Grimson for many fruitful discussions.

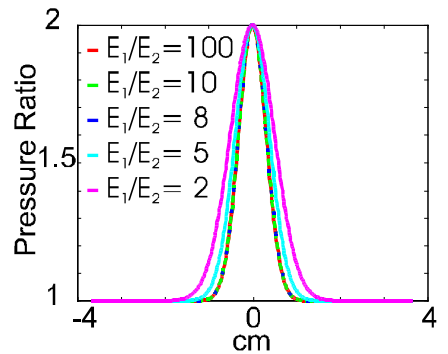

(a)

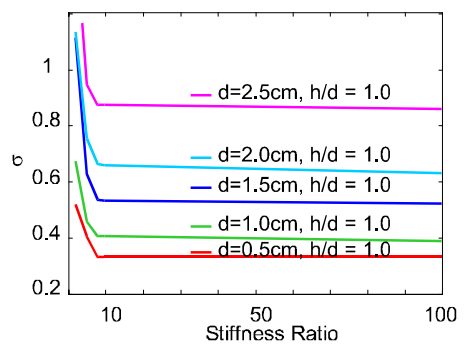

(b)

Fig. 6 - (a) Two-dimensional (FEA derived) tactile maps for a $0.5 \mathrm{~cm}$ diameter lump, 1.0 diameter deep. (b) The width changes by less than 5\% after the stiffness ratio exceeds 8 .

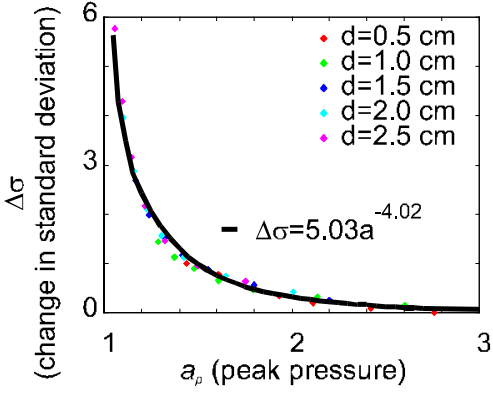

(a)

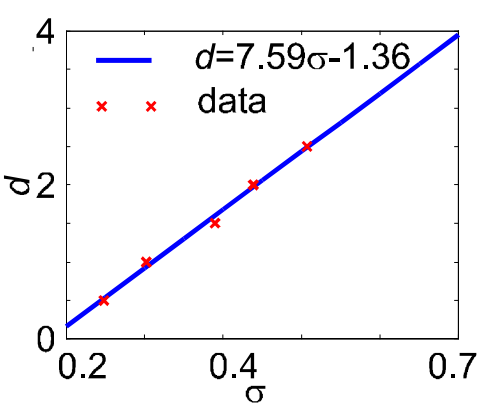

(b)

Fig. 7 - (a) The change in width of the pressure distribution, referenced to 1 diameter in depth, is related to the peak of the pressure distribution by an allotropic relationship. (b) At 1 diameter in depth, the fit parameter $\sigma$ is linearly related to the diameter of the lump, $d$.

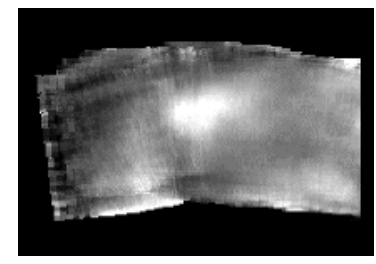

(a)

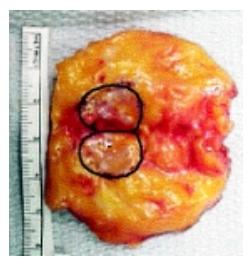

(b)

Fig. 8 - (a) Tactile map of an infiltrating ductal carcinoma. White $=$ highest pressure. The lump is in the center of the map and the sternum is along the lower edge of the map. (b) Photograph of the same mass after surgical excision and bisection parallel to the imaging plane (the black ellipses are the approximate edge of the bisected halves of the tumor). The tactile map size is $15.7 \mathrm{~mm}$ by $13 \mathrm{~mm}$ while its ex vivo palpable size is $16 \mathrm{~mm}$ by $12 \mathrm{~mm}$ 


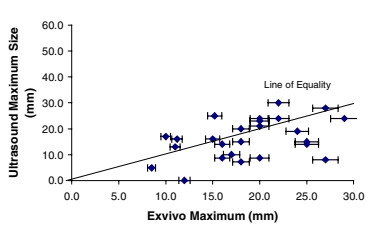

(a)

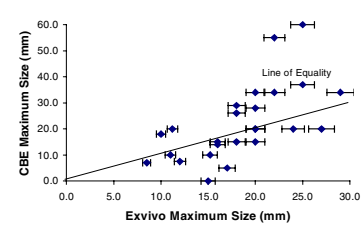

(b)

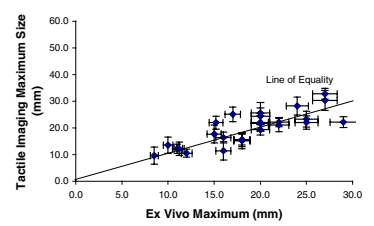

(c)

Fig. 9 - Ultrasound (a), and clinical breast examination (CBE) (b), and tactile imaging (c) for 24 subjects (25 masses). Error bars are $+/-5 \%$ deviation of the ex vivo size measurements (multiple measurements made by multiple examiners) and $+/$ - one standard deviation of the images made of each mass. One ultrasound or CBE examination was performed; no error bars are shown. One mass was not palpable (zero diameter in $\mathrm{CBE}$ ) and one was not visible on the ultrasound (zero diameter).

\section{References}

1. Dixon JM and Mansel RE. Symptoms Assessment and Guidelines for Referral. BMJ. 309;722-726, 1994 Sep. 17.

2. Haagensen CD. Diseases of the Breast, $3^{\text {rd }}$ Edition. p521-527 Philadelphia, Saunders 1986.

3. Lavin PT. Flowerdew G. Studies in variation associated with the measurement of solid tumors. Cancer. 46(5):1286-90, 1980 Sep 1.

4. Dario P, Bergamasco M and Sabatini A. Sensing body structures by an advanced robot system. Proceedings of the 1988 IEEE ICRA. vol.3 (1988) 1758-63.

5. Frei EH, Sollish BD, Yerushalmi S, U.S. Patent \# 4,250,894, (1981)

6. Gentle, C.R. Mammobarography: a possible method of mass breast screening. J. Biomed. Eng., Vol. 10. April 1988.

7. Koganezawa K, Takanishi A, Sugano S eds. Development of Waseda Robot: The Study of Biomechanisms and Kato Laboratory ( $3^{\text {rd }}$ Edition). Waseda University. 1991.

8. Sarvazyan AP. Knowledge-Based Mechanical Imaging. Proceedings of the $10^{\text {th }}$ IEEE Symposium on Computer-Based Medical Systems. p120-125, 1997.

9. Cespedes I, Ophir J, Ponnekanti H and Maklad N. Elastography: elasticity imaging using ultrasound with application to muscle and breast in vivo. Ultrasonic Imaging. (1993) 15(2):73-88.

10. Manduca A, Muthupillai R, Rossman PJ, Greenleaf JF, Ehman RL. Visualization of tissue elasticity by magnetic resonance elastography. Proceedings of Fourth International Conference on Visualization in Biomedical Computing (VBC'96). p.xii+610, 63-8 22-25 Sept. 1996

11. Konofagou E, Dutta P, Ophir J, Cespedes I. Reduction of stress nonuniformities by apodization of compressor displacement in elastography. Ultrasound in Medicine \& Biology. 22(9):1229-36, 1996.

12,21. Johnson, KL. Contact Mechanics. Cambridge University Press. 1987.

13,23. Krouskop TA, Wheeler TM, Kallel F, Garra BS, Hall T, The Elastic Moduli of Breast Prostate Tissues Under Compression, Ultrasonic Imaging. 20:151-159, (1998).

14. Harris JR, Lippman ME, Morrow M and Hellman S. Diseases of the Breast. Lippincott-Raven. 1996.

15. Fung YC. Biomechanics: Mechanical Properties of Living Tissues $2^{\text {nd }}$ Edition. Springer Verlag, NY. 1993.

16. Goodier, J.N. Concentration of stress around spherical and cylindrical inclusions and flaws. Phil. Mag., 7(22):678. 1936.

17. Eshelby, J.D. The determination of the elastic field of an ellipsoidal inclusion and related problems. Proc. R. Soc. Lond., A 241:376-396.

18. Yu, H. Y. and Sanday, S.C. Elastic field in joined semi-infinite solids with an inclusion. Proc. R. Soc. Lond. A 434:521-530. 1991.

19. Fearing, R. S. and Hollerbach, J. M. Basic solid mechanics for tactile sensing. International Journal of Robotics Research, 4(3):40-54, Fall 1985.

20,22,24. Wellman, PS and Howe, RD. The Mechanical Properties of Breast Tissues In Compression. Harvard BioRobotics Laboratory Technical Report \#99003. 1999. 